\title{
Écritures féminines. L'image de la femme à travers le discours dominant sur le féminin
}

Laura LÓPEZ MORALES Universidad Nacional Autónoma de México

Parler de la littérature féminine francophone oblige, d'une part, à parler, ne serait-ce que succintement, de la littérature écrite par les femmes en général. D'autre part, si nous acceptons que l'émergence des lettres dans les divers espaces de la francophonie, ailleurs qu'en Europe, est relativement récente, la place occupée par les écrits féminins l'est encore davantage. Or, pour comprendre le caractère et la portée de la lutte menée par les femmes pour construire leur propre discours, il convient de rappeler les données essentielles du discours dominant qui, depuis la nuit des temps, a réglé officiellement les relations humaines sur la base de la différence des sexes.

Autrement dit, il nous faut préciser que la structure de départ, celle qui articule le discours élaboré par les hommes et qui instaure rapports et rôles entre l'homme et la femme, ne peut pas être contournée par celle-ci au moment de la prise de parole. En effet, la femme doit inévitablement prendre d'abord conscience du message et des mécanismes de ce discours patriarcal pour, ensuite, le subvertir, le démonter jusqu'à ce que qu'elle puisse construire son propre discours et dire ce qu'il en est de son vécu et de ses projets; elle doit, de ce fait, commencer par démolir les mythes que l'on a tissés à son sujet. Les femmes auront donc à lutter contre toute une série de précepts véhiculés par le discours sur le féminin et qui renvoient à des comportements et à des fonctions qu'elle est tenue d'assumer dans la société. Ce discours la décrit par la manière dont elle est censée participer au fonctionnement de la communauté et cette description traduit souvent un type "idéal" de femme que l'homme attend et auquel, bien entendu, elle doit répondre.

Ce discours pose, entre autres, que les comportements féminins correspondent à la dépendance, à la peur, à la faiblesse, à l'insécurité, au manque d'intelligence, d'identité autonome, à l'incapacité... comme s'il s'agissait de traits transmis par le code génétique. Mais à y bien regarder, ce que le discours patriarcal considère comme proprement féminin est très vague et la plupart du temps, en dehors du justificatif purement biologique, il 
renvoie à un ensemble de fonctions, sentiments, qualités, actes... inscrits dans un cadre social, de telle sorte que les différences sociales sont souvent justifiées sur la base des différences naturelles. Ce discours, qui régit encore de nos jours les rapports entre les deux sexes dans les sociétés actuelles, se construit depuis l'Antiquité. La Grèce classique - Hésiode, Homère, Platon, Aristote...- instaure déjà ces différences comme une loi de la nature que la société se doit de respecter. De plus, la femme n'est pas seulement un être "domestique" mais un "objet" d'échanges, de disputes, de conquêtes, d'alliances et de récompenses. Très souvent elle est vue, selon la définition marxiste, comme une "valeur d'usage", objet de production et d'échange.

Le discours sur le "féminin" implique donc les rapports réels entre les êtres qui le produisent; ces rapports renvoient essentiellement à deux fonctions: la reproduction humaine et la reproduction sociale. La première assure la survie de l'espèce sur la planète, l'autre garantit en gros l'apprentissage des normes, des techniques de travail, la socialisation, les formes politiques et religieuses de coexistence, bref, la construction et la transmission de la culture. Ainsi, ce discours situe la femme dans un espace où les actions et les relations qu'elle entretient sont limitées par la seule fonction biologique qui lui est reconnue et assignée, la séparant du discours qui définit le masculin. L'un est "intérieur", l'autre "extérieur".

Le rôle et les fonctions attribués à la femme sont à identifier non seulement dans les allusions explicites que le discours dominant fait, mais on peut les lire aussi dans les silences; c'est-à-dire que, par exemple, dans les récits épiques, les héros des batailles, les protagonistes des exploits, ceux qui s'occupent d'organiser le monde, d'en formuler les lois, sont des hommes. C'est tout un espace de l'action et de la prise de décisions dont la femme est exclue, si cen'est ignorée ou niée. Bref, elle n'a pas d'existence dans la sphère du pouvoir, celui-ci étant l'instance qui élabore les règles du jeu, les lois et les normes, qui dicte les décisions politiques, économiques et familiales. Le rôle qui revient à la femme est donc construit à partir de cette instance du pouvoir et en devient sa justification. Le discours patriarcal en est le témoignage lorsqu'il souligne, comme appartenant à la femme, les attributs suivants: l'obéissance, la soumission, le dévouement, la peur, la pudeur, le mensonge, l'ambivalence, l'ignorance, la dépendance et le silence.

\section{Quelques repères pour l'espace francophone}

Ceci dit, nous ajouterons encore deux mots sur les traits essentiels des nouvelles littératures francophones, d'une part, et, de l'autre, sur les 
caractéristiques générales des écrits féminins contemporains. Pour commencer, l'évolution des lettres francophones est fondamentalement liée à la décolonisation ou du moins aux revendications politiques et culturelles qui cherchent, entre autres, à bâtir et à préserver une identité nationale menacée. Il faut également tenir compte d'un fait qui apparaît comme dénominateur commun à ces littératures, à savoir: la diversité des situations sociolinguistiques découlant des contextes plurilingues et pluriculturels de ces régions. Les écrivains francophones, sans renoncer à la référence à la tradition française, tentent d'exprimer leur propre réalité, les rêves, les richesses et les problèmes de leurs communautés même si dans la plupart des cas ils se servent de la langue de l'ancien colonisateur. Enfin, ces littératures sont nées de l'éclosion d'une conscience politique qui conduit à la conquête d'une identité collective et individuelle longtemps étouffée et sacrifiée. La langue française devient donc instrument de dénonciation, de revendication, mais aussi objet de transgression, de subversion et de création, car le code dominant et imposé autrefois, doit se plier à de nouvelles exigences et exprimer de nouvelles réalités. Les écrivains francophones veulent élaborer leur propre discours, démolir les mythes que le colonisateur avait construits à leur sujet et enfin construire une image authentique et fidèle à ce qu'ils considèrent leur identité. Dans ce sens, il en va de même pour ce qui est de la prise de parole par les femmes en général et doublement, par les femmes francophones.

Pour commencer, nous l'avons déjà avancé, les femmes qui prennent la décision de donner leur vision de la réalité, de faire valoir la manière dont elles se perçoivent et de le dire avec les moyens expressifs qu'elles connaissent, se voient d'abord confrontées au besoin de s'aménager un code plus adapté aux expériences jusqu'alors méconnues ou faussées par le discours patriarcal. Car, comment rendre le vécu de la subordination, de l'écrasement au nom d'une supériorité imposée? Comment revendiquer le droit à la parole à partir d'un statut défini par la soumission et le silence? Comment exprimer les joies et les angoisses de la maternité? Comment, enfin, faire entendre et valoir le discours circulaire de l'affectivité face au discours linéaire de la rationalité masculine?

Nous disions plus haut que pour aspirer à articuler leur propre discours, les femmes ont été obligées de bien saisir d'abord, pour ensuite les démonter, les mécanismes du discours que l'homme avait construit à leur sujet. Elles ont donc dû commencer par se demander qui elles étaient vraiment vis-à-vis de l'image que le discours patriarcal leur assignait. En Afrique, par exemple, des écrivains comme Mongo Béti, Ahmadou Kourouma, Henri Lopès, Ferdinand Oyono et au Maghreb, Rachid Boudjedra, Mohammed Dib ou Driss Chraïbi, plaident d'une certaine manière pour la cause féminine, mais leur 
discours idéalise la femme dans les rôles traditionnels sans pour autant lui céder la parole. La nouvelle génération de femmes doit lutter contre ces mythes.

\section{Des horizons à explorer}

Aussi, la parole des femmes a-t-elle d'abord revêtu un caractère autobiographique qui aspirait à faire valoir la perception et le témoignage d'un vécu du point de vue strictement personnel et féminin, non déduit et assigné selon la perspective mâle. Se situer dans le monde signifie donc poser ses repères individuels et collectifs dans le réseau où l'on est amené à jouer un rôle; reste à savoir si ce rôle correspond aux attentes des autres ou s'il obéit aux besoins et aux appels les plus intimes.

Non sans difficulté, les femmes commencent donc par décrire leur réalité, leur entourage le plus immédiat: famille et cercle social le plus proche, leur être-femme qui se dit à travers le corps - la sexualité et la maternité-, leur affectivité, les sentiments, les émotions, la non-logique rationnelle et, dans le social, la réclusion, la subordination et le silence. La dimension autobiographique est parfois renforcée par la prise de parole à la première personne. C'est le cas, pour n'en citer que quelques exemples, de la Sénégalaise Mariama Bà, de la Guadaloupéenne Simone Schwarz-Bart, de l'Algérienne Assia Djébar, de la Belge Dominique Rolin ou de la Manitobaine Gabrielle Roy. Il faut néanmoins préciser que le récit romanesque, tout en étant nourri de l'expérience de celles qui le construisent, ménage des marges où les données de base peuvent être déplacées ou échangées dans le tissu fictionnel sans porter atteinte contre leur authenticité ou leur légitimité. Nous verrons ainsi se mettre en place des mécanismes de dédoublement qui repartissent entre deux ou plusieurs personnages le vécu, les rêves ou les fantasmes de leur créatrice.

Un autre élément qui précède et vient se greffer à ce besoin de faire valoir son propre témoignage est celui qui pousse la femme à rompre le silence dans lequel elle a toujours été confinée. La parole devient alors arme, soupape. refuge, accomplissement dans une autre forme d'accouchement; l'écriture lui permet de se poser des questions autrefois non formulées, d'exorciser ses fantasmes, d'évacuer le refoulé, enfin de se saisir comme si elle était autre et à la fois elle même. Lorsque le niveau du démontage de son être profond est dépassé, le stade suivant met la femme face à ses rapports avec les autres: couple, enfants, parents, amis et avec d'autres acteurs qui, selon le contexte social, instaurent une dynamique bien particulière dont la femme ne peut se soustraire. Tel est le cas des rapports de pouvoir sur le plan du travail ou, plus 
largement, dans une situation politique de domination. Nombreuses sont les femmes à avoir compris le pouvoir de la parole dans la dénonciation des injustices, dans la revendication des droits individuels et collectifs. Ainsi les Québécoises ou les Algériennes qui, joignant la parole à l'action ont lutté pour la défense et l'identité de leurs peuples. Défense de l'identité féminine, d'une part, mais aussi de l'identité nationale d'une communauté menacée. Il s'agit, pour ces femmes, de s'affirmer pour mieux assumer et assurer le rôle qu'elles entendent jouer dans leur société.

Les Antillaises veulent, quant à elles, récupérer et sauvegarder la mémoire d'un peuple mutilé de son passé et dont la continuité culturelle a souvent été assurée par les femmes. Comme les hommes, ou même davantage, elles ont souffert les avanies d'une histoire qu'elles n'ont pas choisie et l'écriture leur permet à présent de reconstruire ce passé individuel ou collectif et d'édifier un présent plus en accord avec leurs attentes.

Au moment où les peuples commencent à sortir de la longue période de domination dont la durée et les modalités ont varié selon les latitudes, un paradoxe va naître quant au rôle à jouer par les femmes dans l'émergence et le développement de ces nouvelles sociétés. Pour ce qui est des Antilles, Maryse Condé voit très clairement comment, bien avant, l'école coloniale avait renforcé le clivage entre hommes et femmes:

Cette école [qui] était réservée aux garçons [...] a introduit plus qu'un fossé entre "lettrés" et "illettrées", une division radicale entre les deux sexes. Très vite, les femmes moins instruites ont été considérées comme des freins à la nécessaire ascension sociale tandis que s'imposaient des idéaux auxquels elles ne pouvaient d'abord s'identifier. Si aujourd'hui la scolarisation des filles est chose faite dans la majorité des pays, la situation de la femme n'en demeure pas moins fort difficile. De façon contradictoire, on lui demande de rester la détentrice des valeurs traditionnelles et de représenter le rempart contre l'angoissante montée du modernisme alors que la société tout entière est engagée dans la course au progrès. Quand elle cède au vertige général, ce qui est fréquent, on l'accable. ${ }^{1}$

A quelques nuances près, la même situation se produit au Maghreb, en Afrique noire et, peut-être plus attenuée et déguisée, au Québec avant le réveil et la participation éclatante des femmes depuis la Deuxième Guerre Mondiale et ensuite au cours de la Révolution Tranquille des années soixante. En cela, nous reprenons les affirmations de Maryse Condé à propos du rôle

'La Parole des femmes, Paris, L'Harmattan, 1993, pp. 3-4. 
joué par les femmes dans les mouvements de libération nationale; elle en parle en pensant aux Antilles.

Le rôle des femmes au sein des luttes de libération antérieures et postérieures à l'abolition de l'esclavage a été largement occulté. Vivant souvent dans l'Habitation à titre de domestique (cuisinière, bonne d'enfants, lingère), elle a dans bien des cas été responsable des empoisonnements collectifs des maitres et de leur famille, participé aux incendies des plantations, terreur du xville. siècle et marroné en nombre important [...] On peut se demander si la modification du regard que la femme antillaise porte sur elle-même et la dégradation de son statut n'accompagnent pas le progrès de l'urbanisation, la montée de la classe bourgeoise dont les modes de vie sont calqués sur ceux de la "métropole" et la dépendance de plus en plus lourde vis-à-vis des idéaux européens. ${ }^{2}$

Ceci est en partie valable pour le Maghreb et plus particulièrement pour l'Algérie, notamment en ce qui concerne l'anonymat auquel l'histoire a condamné les femmes dont Assia Djebar fait le portrait à travers les siècles et jusqu'à la guerre d'Indépendance.

\section{La maternité}

Point n'est besoin d'insister sur le fait que presque toujours et partout, la seule voie laissée à la femme pour justifier et légitimer sa place dans le monde est la maternité. Or, la littérature ne manquera pas de parler de cette expérience qui peut assumer des valeurs fort différentes, parfois opposées: en tant que source de vie, la maternité transforme la femme en un être presque sacré, elle sera ainsi glorifiée, exaltée, digne de respect et, de fait, elle vit cette expérience comme un accomplissement; du coup, la stérilité est une malédiction. La maternité donne un sens à la vie des femmes; mais ceci est vrai lorsque la maternité est voulue et cherchée; si elle n'est pas désirée, elle est vécue comme une fatalité, car l'exercice de la sexualité implique presque toujours le risque de la grossesse et ce prix est assumé exclusivement par la femme. Sexualité et maternité vont de pair, n'en font qu'un. Simone SchwarzBart exprime fort clairement cette situation quand elle définit les femmes comme "des ventres à crédit" et précise que la femme ne peut pas aimer sans craintes car son "ventre est là pour la trahir".

${ }^{2}$ Ibid., pp. 4-5. 
Mais ne nous y trompons pas, ceci n'est pas seulement le lot des femmes du tiers monde. Chez la romancière belge Dominique Rolin l'annonce de la grossesse est une "découverte contrariante". Dans Trente ans d'amour fou, la protagoniste "refusai[t] d'être un simple mammifère". Pour la Québecoise Anne Hébert, dans Le Torrent, la maternité est vécue comme une expérience indésirable et stigmatisante parce que non voulue; le résultat en est ou le mépris de l'enfant ou la haine aggravé par un sentiment de culpabilité et d'autopunition.

La maternité ne se borne pas au simple fait d'enfanter un "bout de viande", de le faire vivre et de le chérir. II ne suffit pas seulement d'engendrer, de vivre passivement le travail génétique qui se termine par l'accouchement, la fonction biologique entraîne par la suite le devoir de protéger, d'élever et d'assurer le bien-être de la progéniture. Et la femme qui aspire à la plénitude de son rôle de mère doit s'acquitter comme il faut de ce devoir; si jamais elle y fait défaut, elle sera mise au ban de la société.

Par ailleurs, même sans être la mère biologique, la femme est souvent tenue d'en jouer le rôle vis-à-vis de son partenaire ou de ceux qui l'entourent. Il faut admettre qu'elle aime à joeur ce rôle comme il arrive chez les personnages féminins de La Plage d'Ostende de Jacqueline Harpman où cet amour maternel a parfois l'allure d'un sentiment incestueux. Quoi qu'il en soit, sur le plan réel ou symbolique, assumant des valeurs positives ou négatives, la maternité est un repère clé de l'être-femme chez la plupart des écrivaines.

Jusque là, les traits soulignés pourraient faire penser que les écrits de ces femmes obéissent à des mobiles identiques et visent les mêmes cibles. Or, en dépit des convergences peut-être dans la démarche et dans le besoin de faire entendre leur voix de femmes, la réalité impose de faire toutes sortes de nuances en fonction des contextes sociaux d'où chacune de ces écrivaines est amenée à formuler son discours.

Pour les Africaines, par exemple, en dehors de la situation économique et politique précaire qui règne dans la plupart des pays sous-développés. il est des moeurs qui sur le plan social représentent de vraies agressions contre leur intégrité, comme la polygamie et l'excision, pour n'en citer que les plus humiliantes. La littérature en offre beaucoup d'exemples.

La revendication du droit à l'emploi dans les mêmes conditions que les hommes, ou à disposer librement de son corps au sujet des grossesses, est plus le fait des femmes des sociétés industrialisées. Dans les différents pays francophones, la prise de parole en littérature ne renvoie pas aux mêmes réalités. Il est donc dangereux d'appliquer les mêmes critères à des contextes où la population féminine est majoritairement analphabète et à ceux où depuis 
longtemps les femmes ont eu, tant bien que mal, accès à l'éducation et donc aux moyens d'exiger leurs droits.

\section{Caractéristiques du discours féminin}

Parler d'écritures féminines ou d'écritures masculines, ou encore d'écritures androgynes, continue d'être un sujet polémique; on trouvera toujours des contre-exemples aux arguments des uns et des autres. Néanmoins, il y a à propos des écrits de femmes un certain consensus pour ébaucher quelques caractéristiques valables pour l'ensemble, à savoir la dimension autobiographique, la structure non obligatoirement linéaire mais plutôt circulaire ou cyclique, l'expression du corps, des sentiments, des émotions et une attention moins centrée sur l'analyse par opposition à une importance plus grande à l'observation des détails. A part la rupture de la linéarité temporelle et le bouleversement spatial, autres traits associés à l'écriture au féminin seraient la transgression des genres établis, du lexique et de la syntaxe. En témoignent les oeuvres de Rolin, de Bersianik, de Brossard, de Bouraoui. Pour la femme, écrire est souvent un acte subversif.

Ceci dit, la ligne de démarcation entre ce qu'on pourrait considérer comme écritures féminines et écritures masculines n'est ni nette ni évidente et il n'est pas non plus dit que tout ce qui est écrit par une femme est obligatoirement "au féminin" car on peut trouver dans un sexe comme dans l'autre des caractéristiques d'écriture attribuées au sexe opposé, surtout si nous tenons compte que, du point de vue psychanalytique, l'identité sexuelle n'est, à l'origine, qu'une donnée purement biologique, si ce n'est une variable. Les traces de l'identité sexuelle que l'on trouve parfois dans l'écriture des femmes, mais aussi des hommes, apparaissent à leur insu et non pas comme le résultat d'une intention délibérée. Pour certains spécialistes, c'est dans la description que la sexuation du discours peut se manifester car il s'agit là d'un espace où peuvent se tisser des réseaux métaphoriques à travers lesquels affleure la subjectivité qui, comme il a été déjà dit, est sensiblement façonnée par l'identité assumée dans le social.

Ce qui demeure un fait indéniable c'est que dans les lettres, comme dans presque tous les domaines de la vie publique, les femmes affirment de plus en plus leur présence et luttent pour vaincre les résistances qui freinent et entravent leur épanouissement. Les obstacles ne viennent pas seulement de l'extérieur, très souvent ce sont ses propres atavismes que la femme doit surmonter. Le chemin à faire est encore long et plein d'embûches, mais personne ne peut plus contester les acquis et, en tout cas, personne ne peut 
non plus se passer des apports féminins non seulement en littérature mais dans tous les domaines culturels, annulant ainsi le déterminisme évoqué au début qui attribue aux femmes la seule reproduction biologique et à l'homme la responsabilité et le mérite de la prodution et transmission des biens culturels. 